\title{
Improved Boronate Affinity Electrophoresis by Optimization of the Running Buffer for a Single-step Separation of piRNA from Mouse Testis Total RNA
}

\author{
Yusuke Sato, Daijiro Iwasawa, Kuo Ping Hui, Rena Nakagomi, and Seiichi Nishizawa ${ }^{\dagger}$ \\ Department of Chemistry, Graduate School of Science, Tohoku University, Aoba, Sendai 980-8578, Japan
}

\begin{abstract}
Here we examined optimization of the running buffer in boronate affinity electrophoresis for improved separation of PIWI-interacting RNA (piRNA) with 2'-O-methylated ribose in 3'-terminal nucleotide. The use of Good's buffer, such as HEPES, significantly increased the separation efficiency for piRNA over normal RNA with free $3^{\prime}$-terminal ribose, and retained an ability to resolve the difference by at least 4-nucleotide lengths in the target piRNAs. We also demonstrated a single-step separation of piRNA from mouse testis total RNA.
\end{abstract}

Keywords Boronate affinity electrophoresis, piRNA, phenylboronic acid, 2'-O-methylatyion, separation

(Received August 30, 2017; Accepted January 9, 2018; Published May 10, 2018)

\section{Introduction}

PIWI-interacting RNAs (piRNAs), a new class of small noncoding RNAs, are mainly expressed in the germline. ${ }^{1}$ piRNAs are single-stranded RNAs of $26-31$ nucleotide (nt) length, which are slightly longer than the better-known microRNAs $(21-25 n t))^{2,3}$ Their $3^{\prime}$-terminal nucleotides feature 2'-O-ribose methylation by methyltransferase HEN1, which can enhance the piRNA stability. ${ }^{4,5}$ The binding of piRNAs to Piwiclass Argonaute proteins ${ }^{6}$ leads to a silencing of the target complementary messenger RNAs. ${ }^{7,8}$ It has been known that piRNAs play a key role in the protection of germ-cell genomes from the mobile genetic elements, thereby preventing transposon-induced defects in gametogenesis and fertility. ${ }^{9,10}$ Moreover, recent studies uncovered the critical functions of piRNAs in transcriptional regulation and transgenerational inheritance. $^{1}$

$2^{\prime}$-O-Methylation at $3^{\prime}$-terminal nucleotides in piRNAs can be used for discriminating piRNAs from other small RNA species having no $3^{\prime}$-modifications. ${ }^{11,12} \mathrm{NaIO}_{4}$ oxidation and subsequent $\beta$-elimination have been useful for investigating whether the 3 '-terminal nucleotide of target RNAs contains free ribose or not. This reaction specifically occurs for the free cis-diol group at 3'-terminal ribose, which results in the formation of $2^{\prime}$, $3^{\prime}$-cyclic phosphate ends that are one nucleotide shorter. In contrast, piRNAs are tolerant to this chemical treatment due to the presence of $2^{\prime}$-O-methylated ribose. Thus, it can be well analyzed by the standard polyacrylamide electrophoresis. Furthermore, this enables one to construct a piRNA-enriched library by following ligation with an adaptor sequence, since $\mathrm{NaIO}_{4}$-reacted and $\beta$-eliminated RNAs cannot be ligated. ${ }^{13}$ This leads to comprehensive profiling by the deep sequencing method. ${ }^{14}$

Alternatively, Lau et al. recently exploited the boronate

† To whom correspondence should be addressed.

E-mail: nishi@m.tohoku.ac.jp affinity electrophoresis for discriminating piRNAs from other small RNAs in follicle cells from Drosophila ovarium. ${ }^{15}$ The principle is based on specific recognition of boronic acid for the cis-diol group of RNA ribose in gel electrophoresis, as used for the analysis of a $3^{\prime}$-terminal modification of longer RNAs. ${ }^{16,17}$ Thus, RNA species having free $3^{\prime}$-terminal ribose can be captured by boronic acids in the gels, whereas piRNAs with $2^{\prime}$-O-methylation can not. They applied this method to purified RNA samples obtained by immunoprecipitation with PIWI antibody from total RNAs, and successfully separated piRNAs from contaminating RNAs with similar length to piRNAs found in insects. ${ }^{18}$ Isolated piRNAs can be readily subject to a downstream analysis, such as deep-sequencing. ${ }^{15}$ Therefore, this approach benefits from simple and easy operation for piRNA separation compared to the above-mentioned chemical treatment. In this work, we further improved the boronate affinity electrophoresis for piRNA separation based on an examination of the running buffer, demonstrating a single-step piRNA separation from non-purified total RNA.

\section{Experimental}

\section{Reagents}

All of the RNAs and DNAs were custom-synthesized and HPLC-purified by Gene Design Inc. (Osaka, Japan) and Nihon Gene Reserach Laboratoriers Inc. (Sendai, Japan), respectively. Other reagents were of commercially available analytical grade, and were used without further purification. Mouse testis total RNA was purchased from Biochain Institute Inc. (Newark, CA, USA). The concentrations of DNA and RNAs were determined from the absorbance at $260 \mathrm{~nm}$ according to the literature. ${ }^{19}$ Water was deionized ( $\geq 18.0 \mathrm{M} \Omega \mathrm{cm}$ specific resistance) by an Elix 5 UV Water Purification System and a Milli-Q Synthesis A10 system (Millipore Corp., Bedford, MA), followed by filtration through a BioPak filter (Millipore Corp., Bedford, $\mathrm{MA}$ ) in order to remove RNase.

As shown in Table 1, we prepared buffer solutions with 
Table 1 Buffer composition used in this study

\begin{tabular}{ccc}
\hline & \multicolumn{1}{c}{ Composition } & $\mathrm{pH}$ \\
\hline $1 \times$ HEPES & 20 mM HEPES, 5.0 mM sodium acetate, & 8.2 \\
& 1.0 mM EDTA & \\
$1 \times$ MOPS & 20 mM MOPS, 5.0 mM sodium acetate, & 7.9 \\
& 1.0 mM EDTA \\
$1 \times$ TAE & 89 mM Tris, 3.7 mM acetic acid, $4.0 \mathrm{mM}$ & 8.5 \\
& EDTA & \\
$1 \times$ TBE & 89 mM Tris, 7.3 mM boric acid, $4.0 \mathrm{mM}$ & 8.5 \\
& EDTA & \\
\hline
\end{tabular}

standard composition for RNA electrophoresis. Considering the strong binding of phenylboronic acid to diol-containing compounds at higher $\mathrm{pH},{ }^{20}$ HEPES and MOPS buffers were adjusted to $\mathrm{pH} 8.2$ and 7.9, respectively. Both TAE and TBE buffers were adjusted to $\mathrm{pH} 8.5$.

\section{Electrophoresis}

Electrophoresis was performed at room temperature using a mini-slab electrophoresis system (AE6531, ATTO, Tokyo, Japan). $\quad N$-Acroyl-3-aminophenylboronic acid monomer, synthesized according to the literature ${ }^{21}$ was used for preparing boronate affinity gels. ${ }^{16}$ Briefly, $N$-acroyl-3-aminophenylboronic acid monomer was dissolved in a mixture of $40 \%$ acrylamide stock solution (19:1, acrylamide:bis), $7 \mathrm{M}$ urea and $1 \times$ buffer. Then, ammonium persulfate (APS, $0.05 \%$ final concentration) and $N, N, N^{\prime}, N^{\prime}$-tetramethylethylenediamine (TEMED, $0.0005 \%$ final concentration) were added for gel polymerization $\left(37^{\circ} \mathrm{C}\right.$, $90 \mathrm{~min})$. In typical experiments, the resulting gel was prerun at constant power $(30 \mathrm{~mA})$ for $10 \mathrm{~min}$ in $1 \times$ buffer. RNA samples mixed with the RNA loading buffer without ethidium bromide (Wako, Sendai, Japan) were loaded on the prerun gels. Gels were subjected to electrophoresis for $2.5-3 \mathrm{~h}$ at a constant $30 \mathrm{~mA}$ until bromophenol blue in the loading buffer moved $70 \%$ of the gel length. After electrophoresis, the gels were stained by SYBR Gold, and then visualized by LAS-4000 or LAS-1000 mini (Fuji Film, Tokyo, Japan).

\section{Fluorescence measurements}

Fluorescence spectra were measured with a JASCO Model FP-6500 spectrofluorophotometer equipped with a thermoelectrically temperature-controlled cell holder (Japan Spectroscopic Co. Ltd., Tokyo, Japan) using a $3 \times 3 \mathrm{~mm}$ quartz cell. We monitored any changes in the fluorescence intensity of Alizarin red S (ARS) at $614 \mathrm{~nm}$ as a function of the concentration of phenylboronic acid (PBA) in HEPES or TAE buffer at $20^{\circ} \mathrm{C}$. The fluorescence response of ARS for PBA was used for determining the $1: 1$ binding constant $\left(K_{\mathrm{a}}\right)$ using the BenesiHildebrand equation. ${ }^{22}$

\section{Results and Discussion}

Boronate affinity gels for piRNA separation were prepared based on the covalent attachment of phenylboronic acid (PBA) to the gel matrix by copolymerization of $\mathrm{N}$-acroyl-3aminophenylboronic acid monomer in the standard denaturing $15 \%$ polyacrylamide gels. 30nt piRNA-1 that was highly expressed in mouse testis, ${ }^{23}$ was chosen as a target piRNA sequence. As shown in Fig. 1, we used synthetic piRNAs with $2^{\prime}$ - $O$-methylated ribose in 3 'terminal nucleotide (piR1) and with free ribose (umpiR1). Also, we used the corresponding
(A)

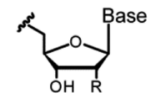

piRNA: $\mathrm{R}=\mathrm{CH}_{3}$
RNA: $\mathrm{R}=\mathrm{OH}^{-}$ RNA: $\mathrm{R}=\mathrm{OH}$
DNA: $\mathrm{R}=\mathrm{H}$

(B)

piR1 : 5'-UGA CAU GAA CAC AGG UGC UCA GAU AGC UU(mU)-3' umpiR1: 5'-UGA CAU GAA CAC AGG UGC UCA GAU AGC UUU-3' piD1 : 5'-TGA CAT GAA CAC AGG TGC TCA GAT AGC TTT-3'

piR2 : 5'-UAC CUU GGA UGU CAC UUC AGA UGC UUC UG(mC)-3' piR3 : 5'-UGA GCA UCC ACU UCU GUG UUU GCU A(mG)-3'

Fig. 1 (A) Structure of 3'-terminal nucleotide in piRNA, RNA and DNA. (B) Sequences of target nucleic acids examined in this study. GenBank accession numbers: piR1, DQ539889; piR2, DQ707092; piR3, DQ715799.

(A)

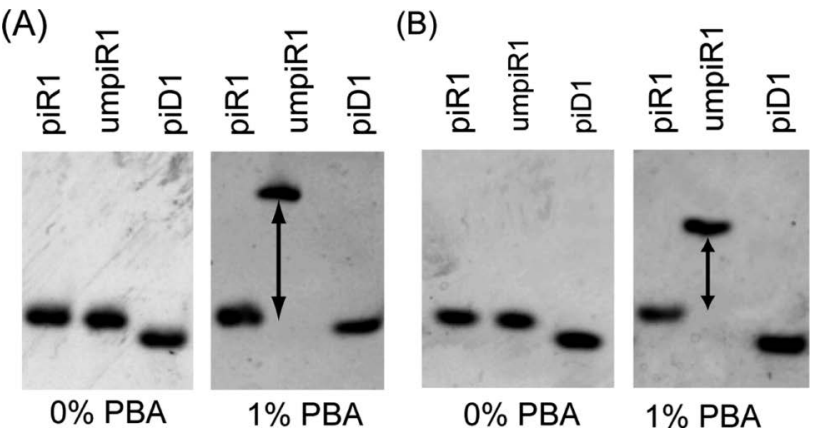

Fig. 2 Electrophoretic mobility of target nucleic acids $(50 \mathrm{ng})$ in the denaturing $15 \%$ polyacrylamide gels in the absence and presence of $1 \%$ PBA using (A) HEPES and (B) TAE running buffer.

DNA sequence, whose ribose at the $3^{\prime}$-terminal nucleobase lacks diol groups (piD1; Fig. 1). In a previous study by Lau et al., tris(hydroxymethyl)aminomethane-acetate EDTA (TAE) buffer ( $\mathrm{pH}$ 8.0) was utilized as a running buffer for piRNA separation based on the boronate affinity electrophoresis. ${ }^{15}$ Likewise, boronate affinity electrophoresis was performed using only the TAE buffer for analyzing 3'-modification of longer RNA. ${ }^{16,17}$ However, tris seemed to interfere with PBA recognition for RNA ribose due to its diol structure, as suggested in the literature. ${ }^{24}$ On the other hand, it was reported that HEPES buffer did not affect the interactions between PBA and diol-containing fluorescent dye (Alizarin red S: ARS). ${ }^{25}$ Hence, we sought to use it and other members of Good's buffer (MOPS: 3-( $N$-morpholino)propanesulfonic acid) for running the boronate affinity electrophoresis (Table 1).

Figure 2A shows the electrophoretic migration of piR1, umpiR1 and piD1 (50 ng) in the absence and presence of $1.0 \%$ PBA in the gels when HEPES buffer was used as a running buffer. The migration distances for each target were shown to be almost the same in gels without PBA, where the relative mobility (RM) value determined as the mobility ratio for piR-1 over umpiR-1 was calculated to be 1.0. This shows that the standard polyacrylamide gel electrophoresis cannot discriminate any difference in the 3 -terminal ribose structure of target nucleic acids. ${ }^{15-17}$ In contrast, piR1 can be clearly resolved from umpiR1 in the presence of PBA (Fig. 2A). Apparently, umpiR1 was significantly retarded in the PBA-containing gel, which yields an effective separation for piR1 against umpiR1 $(\mathrm{RM}=$ 2.8, Fig. 3). Corresponding DNA shows a fast mobility comparable to piR1, because its 3 '-terminal ribose was not captured by the PBA moiety in the gel. This suggests a selective retardation of umpiR1 owing to specific recognition of the PBA moiety for the 3 -terminal free ribose in HEPES buffer. We also observed good separation in the case of MOPS running buffer, 


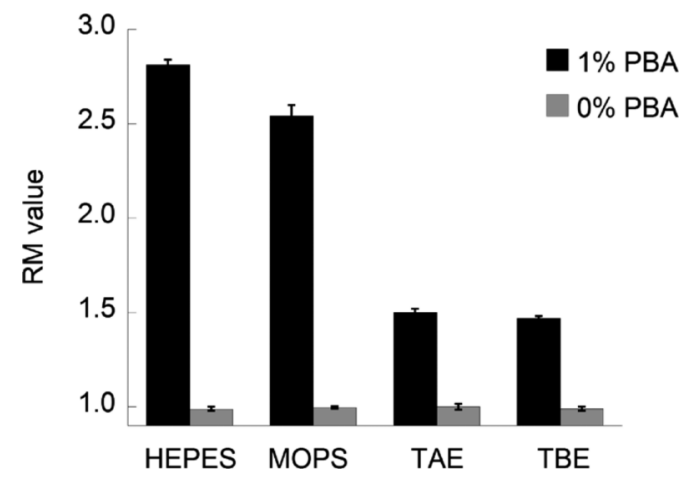

Fig. 3 Summary of the RM values for piR1 against umpiR1 in various running buffers. The error bars are the standard deviations obtained from three independent experiments.

while its separation efficiency was slightly moderate compared to HEPES buffer (RM = 2.5, Fig. 3). On the other hand, when a TAE running buffer was used (Fig. 2B), the RM value for piR1/umpiR1 was remarkably low $(\mathrm{RM}=1.5$, Fig. 3$)$ compared with HEPES and MOPS buffers. Likewise, the use of another tris-containing buffer (TBE: tris-borate EDTA) results in low $\mathrm{RM}$ value $(\mathrm{RM}=1.5$, Fig. 3 ). It is highly likely that the moderate discrimination results from the interference of the tris molecule with PBA recognition of free ribose at the $3^{\prime}$-terminus of umpiR1.

This can be clarified by comparing the binding affinity of PBA to diol-containing fluorescent dye, ARS in HEPES buffer with that in TAE buffer. We carried out fluorescence titration experiments for PBA-ARS interactions (Fig. S1) so that the 1:1 binding constant $\left(K_{\mathrm{a}}\right)$ was obtained using the Benesi-Hildebrand equation (Fig. S2). ${ }^{22}$ PBA binding to ARS in $20 \mathrm{mM}$ HEPES buffer was found to be significantly stronger than that in $20 \mathrm{mM}$ TAE buffer, where the $K_{\mathrm{a}}$ value in HEPES buffer was one order of magnitude higher than that in TAE buffer $\left(K_{\mathrm{a}} / \mathrm{M}^{-1}\right.$ : HEPES, $2500 \pm 98$; TAE, $790 \pm 120(n=3))$. The obtained $K_{\mathrm{a}}$ value in HEPES buffer was in close agreement with the reported value $\left(K_{\mathrm{a}}=2100 \mathrm{M}^{-1}\right.$ in $10 \mathrm{mM}$ HEPES buffer $){ }^{26}$ In addition, the binding affinity of PBA was remarkably reduced when the tris concentration in TAE buffer increased $\left(K_{\mathrm{a}}=280 \pm 88 \mathrm{M}^{-1}\right.$ in $89 \mathrm{mM}$ TAE buffer $(n=3)$ ), whereas the HEPES concentration does not affect the binding affinity $\left(K_{\mathrm{a}}=2700 \pm 78 \mathrm{M}^{-1}\right.$ in $89 \mathrm{mM}$ HEPES buffer $(n=3))$. These results clearly indicate that tris does reduce the recognition ability of the PBA moiety for RNA ribose in gel electrophoresis. Taken together, HEPES buffer provided larger RM values for piR1/umpiR1 (cf. Figs. 2A and 3), which resulted in a significant improvement of the separation ability of boronate affinity electrophoresis.

We found that the target piRNA sequence did not affect its electrophoretic mobility in the PBA-carrying gel. When we examined synthetic piRNA, whose sequence was different from piR-1, but with the same 30nt length (piR-2;27 Fig. 1), its migration was much faster than umpiR1 and comparable to piR1 (Fig. 4). The sequence independence observed here can be explained by stating that the present method relies on the specific recognition of PBA moiety for free ribose of $3^{\prime}$-terminal nucleotide. On the other hand, 26nt piRNA (piR-3; ${ }^{27}$ Fig. 1) showed a larger migration relative to piR-1 and piR-2 in the gel. Apparently, the band of piR-3 can be discriminated from those of 30nt piRNAs, as shown in the migration of the samples mixed with these piRNAs. This clearly shows that the PBAcarrying gel retains the capability to resolve target piRNAs in

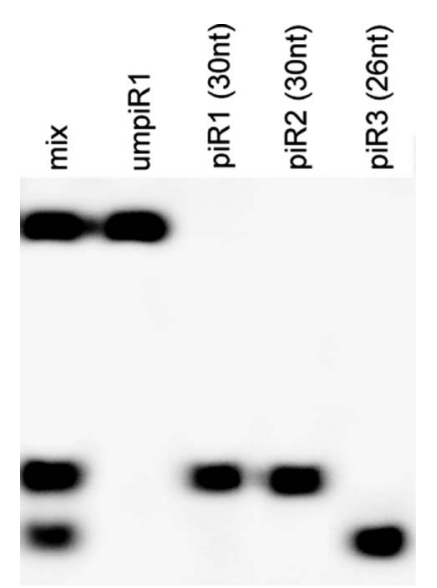

Fig. 4 Electrophoretic mobility of target piRNAs (50 ng) in the denaturing $15 \%$ polyacrylamide gels containing $1 \%$ PBA using $20 \mathrm{mM}$ HEPES running buffer. The mobility of the samples mixed with all piRNAs (50 ng of each piRNA) is also shown.

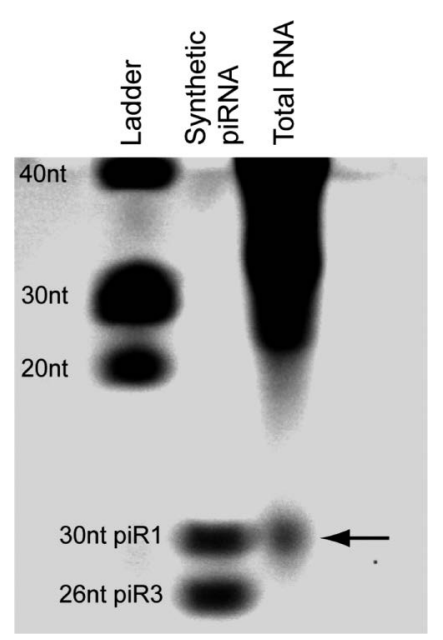

Fig. 5 piRNA separation from mouse testis total RNA $(14.3 \mu \mathrm{g})$ in $1 \%$ PBA-carrying gel. As a control, RNA ladder and synthetic piRNAs (piR1 and piR3) were also applied. The arrow indicates the fraction from piRNAs present in the total RNA.

size, like the standard polyacrylamide gel. Accordingly, the present assay can be useful for the size analysis of target piRNAs, where we can resolve the difference by at least $4 \mathrm{nt}$ length in target piRNAs. As for the size resolution, the ability in HEPES running buffer is comparable to that in TAE buffer (Fig. S3).

Finally, preliminary experiments revealed that the boronate affinity electrophoresis enabled us to separate piRNAs from total RNA in a single step. In contrast to the previous report on purified RNA samples by immunoprecipitation with PIWI antibody, ${ }^{15}$ we examined the direct separation of piRNAs from total mouse testis RNA $(14.3 \mu \mathrm{g})$. As a control, we loaded the synthetic 30nt piR-1 and 26nt piR-3 as well as a small RNA ladder in the same PBA-carrying gel. Note that RNAs in the ladder were retarded because of recognition of the PBA moiety in the gel. As shown in Fig. 5, two fractions were observed. One fraction showed a slower mobility and apparently contained multiple bands, while the one showed a faster mobility. Importantly, these fractions were well resolved. We reasoned 
that in the former one, the bands whose migration are close to 20 - 30nt RNA ladder can be attributed to microRNAs (typically 21 - 25nt) present in the total RNA. MicroRNAs contain free ribose structures at the $3^{\prime}$-terminus, which should be retarded in the PBA-containing gel. Other bands in this fraction are likely to result from other RNA components with a longer length (> 30nt), as previously observed in the standard polyacrylamide gel without PBA moities. ${ }^{11}$ In contrast, the migration of the latter fraction was found to be comparable to synthetic 30nt piR-1. It is highly likely that this comes from piRNAs present in total RNAs considering the length distribution with a peak at 30nt for mouse piRNAs in the database. ${ }^{28}$ The database shows that almost $68 \%$ of the piRNA sequences have 29 - 31nt length, which is consistent with the observed fraction whose migration was very close to $30 \mathrm{nt}$ piR-1. Although it is likely that, due to their lower concentration, other piRNAs with below 28nt or above 32nt length could not be visualized by SYBR gold staining, the results indicate the applicability of the boronate affinity electrophoresis for piRNA separation from mouse testis total RNA in a single step. When we used TAE running buffer, piRNAs could be separated from the total RNA (Fig. S4). However, the separation efficiency was much lower than that in HEPES running buffer. RM values for piRNA fraction in total RNA against 30nt RNA band in the ladder lane were estimated as 2.7 and 1.5 in the case of HEPES and TAE buffer, respectively. These results again emphasize the improved piRNA separation of the boronate affinity electrophoresis by using HEPES running buffer.

In summary, we have demonstrated an improved boronate affinity electrophoresis for the piRNA separation, which allows for a single-step separation of piRNA from mouse testis total RNA. We expect the results obtained here will provide a basis for the design of advanced methods for piRNA separation from various biological sources.

\section{Acknowledgements}

We thank Prof. Minoru Ueda and Dr. Yosuke Takaoka for gel visualization by LAS-4000 imaging system. This work was supported by Grant-in-Aid for Challenging Exploratory Research (No. 15K13716) from Japan Society for the Promotion of Science (JSPS).

\section{Supporting Information}

Supporting information contains the fluorescence response of ARS upon addition to PBA (Fig. S1), Benesi-Hildebrand plot for the binding of PBA to ARS (Fig. S2), electrophoretic mobility of target piRNAs using TAE running buffer (Fig. S3), and piRNA separation from mose testis total RNA using TAE running buffer (Fig. S4). This material is available free of charge on the Web at http://www.jsac.or.jp/analsci/.

\section{References}

1. W-M. Weick and E. A. Miska, Development, 2014, 141, 3458.

2. S. Takalkar, H. Xu, J. Chen, K. Baryeh, W. Qiu, J. X. Zhao, and G. Liu, Anal. Sci., 2016, 32, 617.

3. K. Hasegawa, R. Negishi, M. Matsumoto, M. Yohda, K. Hosokawa, and M. Maeda, Anal. Sci., 2017, 33, 171.

4. M. D. Horwich, C. Li, C. Matranga, V. Vagin, G. Farley, P. Wang, and P. D. Zamore, Curr. Biol., 2007, 17, 1265.

5. K. Saito, Y. Sakaguchi, T. Suzuki, T. Suzuki, H. Siomi, and M. C. Siomi, Genes Dev., 2007, 21, 1603.

6. M. C. Siomi, K. Sato, D. Pezic, and A. A. Aravin, Nat. Rev. Mol. Cell Biol., 2011, 12, 246.

7. J. Brennecke, A. A. Aravin, A. Stark, M. Dus, M. Kellis, R. Sachidanandam, and G. J. Hannon, Cell, 2007, 128, 1089.

8. S. De Fazio, N. Bartonicek, M. Di Giacomo, C. AbreuGoodger, A. Sankar, C. Funaya, C. Antony, P. N. Moreira, A. J. Enright, and D. O'Carroll, Nature, 2011, 480, 259.

9. A. Girard and G. J. Hannon, Trends Cell Biol., 2008, 18, 136.

10. M. C. Siomi and S. Kuramochi-Miyagawa, Curr. Opin. Cell Biol., 2009, 21, 426.

11. Y. Kirino and Z. Mourelatos, Nat. Struct. Mol. Biol., 2007, $14,347$.

12. T. Ohara, Y. Sakaguchi, T. Suzuki, H. Ueda, K. Miyauchi, and T. Suzuki, Nat. Struct. Mol. Biol., 2007, 14, 349.

13. H. Seitz, M. Ghildiyal, and P. D. Zamore, Curr. Biol., 2008, $18,147$.

14. H. Ha, J. Song, S. Wang, A. Kapusta, C. Feschotte, K. C. Chen, and J. Xing, BMC Genomics, 2014, 15, 545.

15. J. A. Matts, Y. Sytnikova, G. Chirn, G. L. Igloi, and N. C. Lau, Methods Mol. Biol., 2014, 1093, 123.

16. G. L. Igloi and H. Kossel, Nucleic Acids Res., 1985, 13, 6881.

17. G. Nubel, F. A. Sorgenfrei, and A. Jaschke, Methods, 2017, $117,14$.

18. N. C. Lau, N. Robine, R. Martin, W. J. Chung, Y. Niki, E. Berezikov, and E. C. Lai, Genome Res., 2009, 19, 1776.

19. J. D. Puglisi and I. Tinocco, Methods Enzymol., 1989, 180, 304.

20. J. Yan, G. Springsteen, S. Deeter, and B. Wang, Tetrahedron, 2004, 60, 11205.

21. D. Roy, J. N. Cambre, and B. S. Sumerlin, Chem. Commun., 2009, 16, 2106.

22. K. A. Conners, "Binding Constants", 1987, Wiley, New York.

23. A. Girard, R. Sachidanandam, G. J. Hannon, and M. A. Carmell, Nature, 2006, 442, 199.

24. J. W. Tomsho and S. J. Benkovic, J. Org. Chem., 2012, 77, 2098.

25. G. Springsteen and B. Wang, Tetrahedron, 2002, 58, 5291.

26. Y. Kubo, T. Ishida, A. Kobayashi, and T. D. James, J. Mater. Chem., 2005, 15, 2889.

27. N. C. Lau, A. G. Seto, J. Kim, S. Kuramochi-Miyagawa, T. Nakano, D. P. Bartel, and R. E. Kingston, Science, 2006, $313,363$.

28. S. S. Lakshmi and S. Agrawal, Nucleic Acids Res., 2008, 36, D173. 